\title{
Incidence and Correlates of Hypoglycemia in Type 2 Diabetes. The Hypos-1 Study
}

Carlo B Giorda ${ }^{1}$, Alessandro Ozzello ${ }^{2}$, Sandro Gentile ${ }^{3}$, Andrea Corsi ${ }^{4}$, Rossella lannarelli ${ }^{5}$, Fabio Baccetti $^{6}$, Giuseppe Lucisano ${ }^{7}$, Antonio Nicolucci ${ }^{7}$ and Maria Chiara Rossi ${ }^{\star}$, On behalf the HYPOS-1 Study Group

${ }^{1}$ Metabolism and Diabetes Unit, ASL TO5, Chieri (TO), Italy

${ }^{2}$ Departmental Service of Diabetology and Metabolic Diseases, ASL TO3, Pinerolo (TO), Italy

${ }^{3}$ Department of Clinical and Experimental Medicine, Second University of Naples, Naples, Italy

${ }^{4}$ Emergency Department, University Hospital St. Martin, Genoa, Italy

${ }^{5}$ Metabolism and Diabetes Unit, San Salvatore Hospital, L'Aquila, Italy

${ }^{6}$ Metabolism and Diabetes Unit, Asl 1, Massa Carrara, Italy

${ }^{7}$ Laboratory of Clinical Epidemiology of Diabetes and Chronic Diseases, Fondazione Mario Negri Sud, Santa Maria Imbaro (CH), Italy

*List of complete study staff in appendix

\section{Abstract}

Background: To evaluate the incidence of severe and symptomatic hypoglycemia in type 2 diabetes and identify their correlates.

Materials and methods: HYPOS-1 is an observational retrospective study. Socio-demographic and clinical characteristics, experience of severe hypoglycemia in the past 12 months and experience of symptomatic hypoglycemia in the past 4 weeks were recorded through a patient questionnaire and a clinical record form. Poisson multivariate models were applied.

Results: Overall, 2023 patients were involved by 18 diabetes clinics. Incidence rate (IR) of severe hypoglycemia was of 0.09 events/person-years; IR of symptomatic hypoglycemia was of 9.30 events/person-years. A minority of patients accounted for the vast majority of severe episodes. The risk of severe hypoglycemia was three times higher in patients with previous severe (Incidence Rate Ratio: 3.38; 95\%confidence interval: $2.47-4.62$ ) and symptomatic hypoglycemia (IRR 3.05 ; $95 \%$ Cl 2.18-4.26); a two-fold risk of severe episodes was associated with basal-bolus insulin regimen (IRR 2.04; $95 \% \mathrm{Cl} 1.24-3.35$ ) as compared with oral therapies not including secretagogues. Glucoselowering therapy (i.e. secretagogues and insulin) and previous severe hypoglycemia represented the strongest predictors of symptomatic hypoglycemia. Female gender was associated with a two-fold risk of severe and a $44 \%$ higher risk of symptomatic episodes as compared to male gender. Age, diabetes duration, HbA1c, neuropathy, retinopathy, overall number of drugs, neoplasms, living status and employment status also increased the risk of severe and/or symptomatic episodes.

Discussion: Hypoglycemia still represents a common acute complication for individuals with type 2 diabetes. Identifying patients at higher risk is a key strategy to reduce the burden of hypoglycemia.

Keywords: Type 2 diabetes; Severe hypoglycemia; Symptomatic hypoglycemia; Risk factors

\section{Introduction}

The goal of diabetes treatment is the maintenance of an adequate metabolic control to prevent or delay the development of complications [1]. However, therapeutic efforts to keep the values of glycated hemoglobin within the recommended target in many cases lead to an increased risk of hypoglycemia [2]. Hypoglycemic episodes, especially the severe ones, are responsible for a relevant clinical, social and economic impact. From the clinical standpoint, physical morbidity of an episode of hypoglycemia ranges from unpleasant symptoms to seizure and coma; rarely, it causes sudden, presumably cardiac arrhythmic death or, if it is profound and prolonged, brain death [3]. Severe hypoglycemia is associated with a higher risk of cardiovascular disease, as confirmed by a recent meta-analysis [4], and dementia $[5,6]$.

Not only severe hypoglycemia but also mild symptomatic hypoglycemia is associated with an increased risk of cardiovascular events, all-cause hospitalization, and all-cause mortality [7].

From the social point of view, several studies have investigated the impact of hypoglycemia on quality of life, suggesting a negative impact on mental well-being and on the overall self-perception of general health status $[8,9]$. Hypoglycemia can disrupt many everyday activities such as driving, work performance and recreational pursuits [10]. In addition, fear of hypoglycemia prevails over fear of long-term complications and is often responsible for poor adherence to therapy or incorrect behaviors of compensation; the latter in turn are responsible for worse metabolic control $[11,12]$.

Hypoglycemia is also an important cause of direct and indirect costs. In the course of a year, up to one third of the subjects with type 1 diabetes of long duration and a fifth of those with type 2 diabetes treated with insulin have at least one episode of severe hypoglycemia, which often requires hospitalization $[13,14]$. In addition to the direct costs, which have been estimated to exceed 3000 euros per episode in several European countries, indirect costs related to lost productivity and absence from work are also relevant $[15,16]$.

*Corresponding author: Maria Chiara Rossi, Laboratory of Clinical Epidemiology of Diabetes and Chronic Diseases, Fondazione Mario Negri Sud, Via Nazionale 66030 S. Maria Imbaro (CH), Italy, Tel: +39 0872 570266; Fax +39 0872570263 E-mail: mrossi@negrisud.it

Received February 14, 2014; Accepted March 18, 2014; Published March 23 2014

Citation: Giorda CB, Ozzello A, Gentile S, Corsi A, lannarelli R, et al. (2014) Incidence and Correlates of Hypoglycemia in Type 2 Diabetes. The Hypos-1 Study. J Diabetes Metab 5: 344. doi:10.4172/2155-6156.1000344

Copyright: (c) 2014 Giorda CB, et al. This is an open-access article distributed under the terms of the Creative Commons Attribution License, which permits unrestricted use, distribution, and reproduction in any medium, provided the original author and source are credited. 
Improvement of the current care requires an accurate evaluation of the burden of hypoglycemia for the healthcare system and the identification of modifiable factors related to an increased risk of hypoglycemia.

In line with these purposes, the Italian Association of Clinical Diabetologists (Associazione Medici Diabetologi - AMD) has promoted the HYPOS-1 initiative, aiming to quantify the incidence of severe and symptomatic hypoglycemia and their determinants in a large cohort of patients with type 2 diabetes.

\section{Materials and Methods}

\section{Italian healthcare system}

In Italy, all citizens are covered by a government health insurance. Primary care for diabetes is provided by general practitioner (GP) and diabetes outpatient clinics (DOCs). Patients can choose one of the two ways of access to health care system or, more frequently, are referred to DOCs by their GPs.

\section{Study design and data collection}

HYPOS-1 is an observational retrospective study involving individuals with type 2 diabetes routinely referred to DOCs.

Eligibility criteria were: male or female gender, age $>=18$ years, diabetes diagnosis at least 1 year before the recruitment in the study, diabetes treatment based on lifestyle intervention or pharmacologic therapies unchanged since at least 1 year, signed informed consent. Patients were not eligible for the study only if they were judged by the investigator as unable to fill in the questionnaire and understand its content.

On the occasion of a routine visit, a sample of consecutive cases has been asked to fill in the HYPOS-1 patient questionnaire. Sampling was stratified by treatment according to the following proportions, reflecting the distribution of the population with type 2 diabetes attending DOCs [17]:

\section{$60 \%$ treated with oral hypoglycemic agent/GLP-1 receptor agonists (OHA) \\ - $\quad 15 \%$ treated with OHA+insulin \\ - $\quad 15 \%$ treated with insulin \\ - $\quad 10 \%$ treated with lifestyle interventions only}

The patient questionnaire investigated the following aspects: sociodemographic and clinical characteristics, including age, gender, school education, employment status, living status, marital status, whether the patient had to care for other people, number of daily medications other than glucose lowering ones, experience of severe hypoglycemia episodes in the past 12 months, and experience of symptomatic hypoglycemia episodes in the past 4 weeks.

Severe hypoglycemia was defined as an episode of hypoglycemia that led to unconsciousness or requiring intervention of a third person; symptomatic hypoglycemia was defined as onset of one or more symptoms including palpitations, tremors, sweating, difficulty concentrating, dizziness, hunger, blurred vision, sense of confusion, difficulty in movement, resolved with the ingestion of sugar, food or sugary drinks. The occurrence of severe hypoglycemic episodes was further confirmed by clinical documentation.

An additional web-based clinical record form (eCRF) was filled in by investigators to collect the following clinical data: weight, height, year of diabetes diagnosis, last value of $\mathrm{HbAlc}$, last value of serum creatinine, presence of diabetes complications (cardiac/cerebrovascular events, lower limb complications, retinopathy, nephropathy, sensorymotor neuropathy, autonomic neuropathy) and chronic conditions, including neoplasms, glucose lowering therapy, use of self-monitoring blood-glucose (SMBG), antihypertensive treatments, and lipidlowering treatments.

Glomerular filtration rate (GFR) was calculated according to the Chronic Kidney Disease Epidemiology Collaboration (CKD-Epi) formula.

Patients were identified by a unique ID number, also used as a key linkage to merge data from patient questionnaires and eCRFs.

Data were analyzed anonymously. The study was approved by the local ethics committees of all participating centers.

\section{Statistical analyses}

Sample size estimation: Assuming an incidence rate of total hypoglycemic events of 0.15 per person-years, a total sample of 2024 patients allows to identify with a statistical power of $80 \%($ alpha $=0.05)$ the risk factors that have a prevalence of at least $20 \%$ and are associated with a higher probability of at least $50 \%$ to have the event. The estimate is based on multivariate Poisson model, assuming that among the covariates in the model there is a R2 of 0.10 [18].

Data analysis: Patients'characteristics according to the presence of severe and symptomatic hypoglycemia are expressed as means and their standard deviations or frequencies. Between-group comparisons are based on the chi-square test for categorical variables and MannWhitney U-test for continuous variables. Univariate associations between patient characteristics and risk of severe and symptomatic hypoglycemia were also expressed as relative risks and their $95 \%$ confidence intervals.

Incidence rates (IRs) of severe and symptomatic hypoglycemia were evaluated through Poisson regression models and expressed as number of events/person-years. Factors associated with the risk of severe and symptomatic hypoglycemia were evaluated through Poisson multivariate models. Results are expressed as incidence rate ratios (IRRs) and their $95 \%$ confidence intervals ( $95 \% \mathrm{CI}$ ). Covariates included in the models were: age, gender, BMI, duration of diabetes, glucose lowering therapy schemes, HbAlc, GFR, ACE-Inhibitors and beta-blockers treatment (classes of drugs associated with reduced awareness) $[19,20]$, previous severe hypoglycemia (before the past 12 months), retinopathy, autonomic neuropathy, sensory-motor neuropathy, neoplasms, number of drugs other than glucose lowering ones, employment status, level of school education, marital status, living status, taking care of other people. Symptomatic hypoglycemia was tested as a predictor of severe hypoglycemia.

\section{Results}

Overall, 18 DOCs enrolled 2023 patients with type 2 diabetes, of whom 202 (10.0\%) were treated with lifestyle intervention, 1212 (59.9\%) were treated with OHA, $306(15.1 \%)$ with OHA+insulin and 303 (15.0\%) with insulin alone. Patients had the following characteristics: $55.3 \%$ males, mean age of $66.3 \pm 10.2$ years, diabetes duration of $11.5 \pm 8.9$ years, HbAlc $7.1 \pm 1.2 \%$. Clinical and socio-demographic characteristics of the study sample according to the occurrence of severe and symptomatic hypoglycemic episodes are shown in Table 1.

Information on severe hypoglycemic episodes was available for 
Citation: Giorda CB, Ozzello A, Gentile S, Corsi A, lannarelli R, et al. (2014) Incidence and Correlates of Hypoglycemia in Type 2 Diabetes. The Hypos-1 Study. J Diabetes Metab 5: 344. doi:10.4172/2155-6156.1000344

Page 3 of 8

\begin{tabular}{|c|c|c|c|c|c|c|c|c|c|}
\hline & All & Severe NO & Severe YES & $\mathbf{p}^{*}$ & $\begin{array}{c}R^{* *} \\
(95 \% \mathrm{Cl})\end{array}$ & $\begin{array}{c}\text { Symptomatic } \\
\text { NO }\end{array}$ & $\begin{array}{c}\text { Symptomatic } \\
\text { YES }\end{array}$ & $\mathbf{p}^{*}$ & $\begin{array}{c}\mathrm{RR}^{* *} \\
(95 \% \mathrm{Cl})\end{array}$ \\
\hline N & 2023 & 1791 & 88 & & & 1442 & 564 & & \\
\hline Male (\%) & 55.3 & 54.9 & 50.0 & 0.36 & $\begin{array}{c}0.84 \\
(0.56-1.26)\end{array}$ & 57.4 & 50.3 & 0.004 & $\begin{array}{c}0.81 \\
(0.71-0.93)\end{array}$ \\
\hline Age (years) & $66.3 \pm 10.2$ & $66.3 \pm 10.1$ & $68.8 \pm 10.7$ & 0.01 & - & $66.3 \pm 9.9$ & $66.4 \pm 10.7$ & 0.40 & - \\
\hline BMI $\left(\mathrm{Kg} / \mathrm{m}^{2}\right)$ & $29.7 \pm 5.7$ & $29.7 \pm 5.8$ & $30.4 \pm 6.0$ & 0.18 & - & $29.7 \pm 5.6$ & $29.9 \pm 6.1$ & 0.90 & - \\
\hline Duration of diabetes (years) & $11.5 \pm 8.9$ & $11.3 \pm 8.7$ & $16.1 \pm 11.7$ & 0.0001 & - & $10.6 \pm 8.5$ & $13.7 \pm 9.4$ & $<0.0001$ & - \\
\hline $\mathrm{HbA} 1 \mathrm{c}(\%)$ & $7.1 \pm 1.2$ & $7.1 \pm 1.2$ & $7.5 \pm 1.4$ & 0.002 & - & $7.1 \pm 1.2$ & $7.2 \pm 1.1$ & 0.0003 & - \\
\hline GFR < 60 mL/min (\%) & 25.6 & 20.5 & 24.4 & 0.39 & $\begin{array}{c}1.24 \\
(0.74-1.98)\end{array}$ & 18.9 & 25.7 & 0.001 & $\begin{array}{c}1.32 \\
(1.11-1.55)\end{array}$ \\
\hline \multicolumn{10}{|l|}{ Diabetes complications (\%) } \\
\hline Cardiac/cerebrovascular & 17.8 & 17.5 & 19.3 & 0.67 & $\begin{array}{c}1.12 \\
(0.64-1.82)\end{array}$ & 16.4 & 21.5 & 0.008 & $\begin{array}{c}1.25 \\
(1.06-1.47)\end{array}$ \\
\hline Lower limb complications & 8.4 & 8.0 & 10.2 & 0.46 & $\begin{array}{c}1.29 \\
(0.61-2.37)\end{array}$ & 6.9 & 12.4 & $<0.0001$ & $\begin{array}{c}1.54 \\
(1.25-1.85)\end{array}$ \\
\hline Retinopathy & 21.0 & 20.4 & 40.9 & $<0.0001$ & $\begin{array}{c}2.54 \\
(1.67-3.82)\end{array}$ & 16.8 & 31.6 & $<0.0001$ & $\begin{array}{c}1.74 \\
(1.51-2.00)\end{array}$ \\
\hline Nefropathy & 18.4 & 17.9 & 25.0 & 0.09 & $\begin{array}{c}1.49 \\
(0.91-2.34)\end{array}$ & 16.4 & 24.1 & $<0.0001$ & $\begin{array}{c}1.40 \\
(1.19-1.63)\end{array}$ \\
\hline Sensory-motor neuropathy & 11.9 & 11.6 & 25.6 & 0.0001 & $\begin{array}{c}2.46 \\
(1.51-3.84)\end{array}$ & 8.8 & 19.9 & $<0.0001$ & $\begin{array}{c}1.84 \\
(1.56-2.14)\end{array}$ \\
\hline Autonomic neuropathy & 5.4 & 5.4 & 13.8 & 0.001 & $\begin{array}{c}2.61 \\
(1.38-4.44)\end{array}$ & 3.9 & 9.0 & $<0.0001$ & $\begin{array}{c}1.75 \\
(1.39-2.14)\end{array}$ \\
\hline Neoplasms (\%) & 4.5 & 4.4 & 9.2 & 0.04 & $\begin{array}{c}2.08 \\
(0.95-3.89)\end{array}$ & 2.0 & 3.2 & 0.11 & $\begin{array}{c}1.23 \\
(0.89-1.61)\end{array}$ \\
\hline \multicolumn{10}{|l|}{ Glucose lowering pharmacologic treatments (\%) } \\
\hline Metformin & 60.5 & 61.0 & 48.9 & 0.02 & $\begin{array}{c}0.63 \\
(0.42-0.94)\end{array}$ & 63.3 & 53.7 & $<0.0001$ & $\begin{array}{c}0.75 \\
(0.66-0.87)\end{array}$ \\
\hline Sulphanylureas & 21.0 & 20.9 & 18.2 & 0.53 & $\begin{array}{c}0.85 \\
(0.48-1.40)\end{array}$ & 21.4 & 20.0 & 0.51 & $\begin{array}{c}0.94 \\
(0.79-1.12)\end{array}$ \\
\hline Glinides & 12.0 & 12.0 & 13.6 & 0.63 & $\begin{array}{c}1.15 \\
(0.61-2.00)\end{array}$ & 10.8 & 15.3 & 0.006 & $\begin{array}{c}1.31 \\
(1.08-1.57)\end{array}$ \\
\hline Acarbose & 4.2 & 4.0 & 3.4 & 0.79 & $\begin{array}{c}0.86 \\
(0.21-2.22)\end{array}$ & 4.3 & 3.9 & 0.69 & $\begin{array}{c}0.93 \\
(0.62-1.30)\end{array}$ \\
\hline Glitazones & 5.4 & 5.1 & 5.7 & 0.82 & $\begin{array}{c}1.11 \\
(0.40-2.39)\end{array}$ & 5.9 & 4.1 & 0.11 & $\begin{array}{c}0.75 \\
(0.50-1.05)\end{array}$ \\
\hline DPP-IV inhibitors & 16.7 & 17.2 & 9.1 & 0.05 & $\begin{array}{c}0.49 \\
(0.22-0.95)\end{array}$ & 17.6 & 14.9 & 0.15 & $\begin{array}{c}0.87 \\
(0.70-1.05)\end{array}$ \\
\hline GLP-1 receptor agonists & 4.8 & 4.8 & 8.0 & 0.17 & $\begin{array}{c}1.68 \\
(0.72-3.26)\end{array}$ & 5.3 & 3.9 & 0.20 & $\begin{array}{c}0.79 \\
(0.52-1.11)\end{array}$ \\
\hline Human regular insulin & 0.4 & 0.4 & 0.0 & 0.56 & $\begin{array}{c}0.00 \\
(0.00-0.00)\end{array}$ & 0.4 & 0.4 & 0.98 & $\begin{array}{c}1.02 \\
(0.19-2.32)\end{array}$ \\
\hline Short-acting insulin analogues & 21.6 & 20.4 & 52.3 & $<0.0001$ & $\begin{array}{c}3.90 \\
(2.60-5.86)\end{array}$ & 14.4 & 39.9 & $<0.0001$ & $\begin{array}{c}2.42 \\
(2.12-2.76)\end{array}$ \\
\hline Basal insulin analogues & 25.4 & 24.2 & 51.1 & $<0.0001$ & $\begin{array}{c}3.06 \\
(2.04-4.60)\end{array}$ & 19.3 & 40.9 & $<0.0001$ & $\begin{array}{c}2.03 \\
(1.77-2.32)\end{array}$ \\
\hline Premix insulin & 4.2 & 4.3 & 8.0 & 0.11 & $\begin{array}{c}1.85 \\
(0.79-3.58)\end{array}$ & 3.1 & 7.1 & $<0.0001$ & $\begin{array}{c}1.75 \\
(1.35-2.17)\end{array}$ \\
\hline Glucose lowering therapy schemes (\%) & & & & $<0.0001$ & & & & $<0.0001$ & \\
\hline $\mathrm{OHA}$ (combination with secretagogues) & 33.5 & 34.1 & 15.9 & & 1.00 & 38.4 & 20.7 & & 1.00 \\
\hline $\mathrm{OHA}$ (combination without secretagogues) & 26.4 & 26.4 & 22.7 & & $\begin{array}{c}1.81 \\
(0.93-3.63)\end{array}$ & 25.9 & 28.0 & & $\begin{array}{c}1.70 \\
(1.38-2.11)\end{array}$ \\
\hline Insulin (basal oral) & 6.7 & 6.6 & 8.0 & & $\begin{array}{c}2.48 \\
(0.96-5.83)\end{array}$ & 6.6 & 6.9 & & $\begin{array}{c}1.65 \\
(1.19-2.23)\end{array}$ \\
\hline Insulin (basal bolus) & 15.6 & 14.5 & 40.9 & & $\begin{array}{c}5.45 \\
(3.06-10.30)\end{array}$ & 10.5 & 28.6 & & $\begin{array}{c}2.95 \\
(2.43-3.60)\end{array}$ \\
\hline Insulin (Other schemes) & 7.9 & 8.0 & 12.5 & & $\begin{array}{c}3.21 \\
(1.45-6.92)\end{array}$ & 5.8 & 12.9 & & $\begin{array}{c}2.68 \\
(2.11-3.38)\end{array}$ \\
\hline Diet & 10.0 & 10.5 & 0 & & - & 12.8 & 2.8 & & - \\
\hline SMBG (\%) & 86.9 & 86.3 & 99.0 & $<0.001$ & $\begin{array}{c}13.10 \\
(2.94-230.38)\end{array}$ & 83.8 & 95.2 & $<0.0001$ & $\begin{array}{c}2.96 \\
(2.10-4.37)\end{array}$ \\
\hline Number of drugs other than glucose lowering ones & $3.3 \pm 2.6$ & $3.3 \pm 2.6$ & $3.8 \pm 2.7$ & 0.03 & & $3.1 \pm 2.6$ & $3.7 \pm 2.7$ & $<0.0001$ & - \\
\hline Antihypertensive treatments $(\%)$ & 70.7 & 70.5 & 70.4 & 0.99 & $\begin{array}{c}1.00 \\
(0.65-1.59)\end{array}$ & 70.6 & 71.9 & 0.55 & $\begin{array}{c}1.05 \\
(0.90-1.23)\end{array}$ \\
\hline
\end{tabular}


Citation: Giorda CB, Ozzello A, Gentile S, Corsi A, lannarelli R, et al. (2014) Incidence and Correlates of Hypoglycemia in Type 2 Diabetes. The Hypos-1 Study. J Diabetes Metab 5: 344. doi:10.4172/2155-6156.1000344

Page 4 of 8

\begin{tabular}{|c|c|c|c|c|c|c|c|c|c|}
\hline ACE-Inhibitors (\%) & 29.1 & 29.3 & 28.4 & 0.86 & $\begin{array}{c}0.96 \\
(0.60-1.49)\end{array}$ & 28.5 & 31.0 & 0.26 & $\begin{array}{c}1.09 \\
(0.94-1.26)\end{array}$ \\
\hline Beta-blockers (\%) & 24.6 & 24.1 & 33.0 & 0.06 & $\begin{array}{c}1.51 \\
(0.97-2.31)\end{array}$ & 23.9 & 26.8 & 0.17 & $\begin{array}{c}1.12 \\
(0.95-1.30)\end{array}$ \\
\hline Lipid-lowering treatments (\%) & 56.4 & 56.8 & 56.8 & 1.00 & $\begin{array}{c}1.00 \\
(0.66-1.52)\end{array}$ & 55.6 & 59.1 & 0.16 & $\begin{array}{c}1.11 \\
(0.96-1.28)\end{array}$ \\
\hline Employment status (\%): & & & & 0.63 & & & & 0.94 & \\
\hline Employed & 20.9 & 20.6 & 18.8 & & 1.00 & 21.1 & 20.5 & & 1.00 \\
\hline Unemployed & 19.1 & 19.7 & 16.5 & & $\begin{array}{c}1.09 \\
(0.54-2.23)\end{array}$ & 18.9 & 19.4 & & $\begin{array}{c}0.96 \\
(0.77-1.20)\end{array}$ \\
\hline Retired & 60.1 & 59.7 & 64.7 & & $\begin{array}{c}1.28 \\
(0.75-2.37)\end{array}$ & 60.1 & 60.1 & & $\begin{array}{c}0.98 \\
(0.82-1.18)\end{array}$ \\
\hline Highest level of school education (\%): & & & & 0.11 & & & & 0.20 & \\
\hline Primary school & 40.3 & 40.3 & 52.3 & & 1.00 & 39.2 & 43.6 & & 1.00 \\
\hline Middle school & 30.6 & 30.3 & 28.4 & & $\begin{array}{c}0.73 \\
(0.45-1.17)\end{array}$ & 31.7 & 27.5 & & $\begin{array}{c}0.84 \\
(0.70-0.99)\end{array}$ \\
\hline High school & 22.8 & 23.3 & 14.8 & & $\begin{array}{c}0.50 \\
(0.26-0.89)\end{array}$ & 22.9 & 22.1 & & $\begin{array}{c}0.90 \\
(0.75-1.08)\end{array}$ \\
\hline University & 6.3 & 6.1 & 4.6 & & $\begin{array}{c}0.59 \\
(0.18-1.42)\end{array}$ & 6.1 & 6.8 & & $\begin{array}{c}1.00 \\
(0.73-1.30)\end{array}$ \\
\hline Living status (\%): & & & & 0.89 & & & & 0.49 & \\
\hline Spouse/sons & 77.8 & 78.0 & 76.1 & & 1.00 & 78.6 & 75.5 & & 1.00 \\
\hline Alone & 15.8 & 15.7 & 18.2 & & $\begin{array}{c}1.18 \\
(0.67-1.95)\end{array}$ & 15.4 & 17.2 & & $\begin{array}{c}1.11 \\
(0.92-1.33)\end{array}$ \\
\hline Other family members & 4.3 & 4.5 & 3.4 & & $\begin{array}{c}0.79 \\
(0.20-2.07)\end{array}$ & 4.1 & 5.1 & & $\begin{array}{c}1.21 \\
(0.86-1.61)\end{array}$ \\
\hline Other & 2.1 & 1.9 & 2.3 & & $\begin{array}{c}1.20 \\
(0.20-3.62)\end{array}$ & 2.0 & 2.1 & & $\begin{array}{c}1.06 \\
(0.61-1.62)\end{array}$ \\
\hline Marital status (\%): & & & & 0.65 & & & & 0.08 & \\
\hline Not married & 7.4 & 7.3 & 4.6 & & 1.00 & 7.4 & 7.2 & & 1.00 \\
\hline Married/cohabitant & 75.2 & 75.5 & 78.4 & & $\begin{array}{c}1.64 \\
(0.69-5.32)\end{array}$ & 76.5 & 71.9 & & $\begin{array}{c}0.98 \\
(0.76-1.32)\end{array}$ \\
\hline Separated/divorced & 4.3 & 4.2 & 5.7 & & $\begin{array}{c}2.12 \\
(0.58-8.35)\end{array}$ & 3.9 & 5.4 & & $\begin{array}{c}1.27 \\
(0.85-1.88)\end{array}$ \\
\hline Widower & 13.1 & 13.0 & 11.4 & & $\begin{array}{c}1.38 \\
(0.47-4.97)\end{array}$ & 12.1 & 15.6 & & $\begin{array}{c}1.22 \\
(0.90-1.69)\end{array}$ \\
\hline Taking care of other people (\%) & 37.3 & 37.2 & 29.6 & 0.15 & $\begin{array}{c}0.72 \\
(0.45-1.11)\end{array}$ & 36.1 & 40.0 & 0.11 & $\begin{array}{c}1.12 \\
(0.97-1.30)\end{array}$ \\
\hline $\begin{array}{l}\text { Previous experience of severe hypoglycemia } \\
\text { (before the last } 12 \text { months) }(\%)\end{array}$ & 10.0 & 9.1 & 36.4 & $<0.0001$ & $\begin{array}{c}4.93 \\
(3.24-7.37)\end{array}$ & 6.3 & 19.3 & $<0.0001$ & $\begin{array}{c}2.16 \\
(1.85-2.50)\end{array}$ \\
\hline
\end{tabular}

Data are expressed as means and their standard deviations or frequencies.

* chi-square test for categorical variables and Mann-Whitney U-test for continuous variables. ${ }^{* *}$ Relative risk and $95 \%$ confidence intervals.

Table 1: Clinical and socio-demographic characteristics according to the experience of severe and/or symptomatic hypoglycemic episodes

1879 patients (93.0\%), while data on symptomatic hypoglycemic episodes were reported by 2006 patients (99.2\%). Patients experiencing at least one severe hypoglycemic episode in the past 12 months were 88 , representing $4.7 \%$ of the sample, while those experiencing at least one symptomatic hypoglycemic episode in the past four weeks were 564, representing $28.1 \%$ of the sample. Among patients with severe hypoglycemia, the number of episodes ranged from 1 to 18 , with $57.3 \%$ reporting 1 episode, $24.7 \%$ reporting 2 episodes, and $18.0 \%$ reporting 3 or more episodes.

Among patients with symptomatic hypoglycemia, the number of episodes ranged from 1 to 30 (1-3 episodes for $77.9 \%$ of the cases, $4-9$ episodes for $18.0 \%$ of the cases, and 10 or more episodes for $4.1 \%$ ).

Among patients with symptomatic hypoglycemia, $89.7 \%$ reported diurnal episodes and $23.4 \%$ reported nocturnal episodes.

Overall, incidence rate of severe hypoglycemia was of 0.09 events/ person-years, while incidence rate of symptomatic hypoglycemia was of 9.30 events/person-years. Incidence rates according to gender, age, diabetes duration history of cardiac/cerebrovascular disease, and glucose lowering therapy scheme are shown in Table 2 . Significantly higher incidence rates of severe and/or symptomatic hypoglycemia were found in female vs. male individuals, in older patients, in patients with a recent diabetes diagnosis (duration $<=2$ years), and in the presence of cardiac/cerebrovascular complications. Moreover, incidence markedly varied according to the classes of glucose lowering treatments, being more pronounced in patients treated with intensive therapy regimens; in particular, patients treated with secretagogues had a doubled risk of severe episode as compared with those treated with other OHAs, while patients on basal-bolus regimens had an almost 7 fold increased likelihood to report a severe episode and a 3-fold increased likelihood to report a symptomatic episode as compared to patients treated with OHAs schemes not including secretagogues.

Results of the fully adjusted multivariate analyses are shown in Table 3. Table 3a shows correlates of severe hypoglycemia and Table $3 b$ shows correlates of symptomatic hypoglycemia. 
Citation: Giorda CB, Ozzello A, Gentile S, Corsi A, lannarelli R, et al. (2014) Incidence and Correlates of Hypoglycemia in Type 2 Diabetes. The Hypos-1 Study. J Diabetes Metab 5: 344. doi:10.4172/2155-6156.1000344

Page 5 of 8

\begin{tabular}{|c|c|c|c|c|}
\hline & \multicolumn{2}{|c|}{ Severe hypoglycemia } & \multicolumn{2}{|c|}{ Symptomatic hypoglycemia } \\
\hline & $\begin{array}{c}\text { IR } \\
(95 \% \mathrm{Cl})\end{array}$ & $\begin{array}{c}\text { IRR } \\
(95 \% \mathrm{Cl})\end{array}$ & $\begin{array}{c}\text { IR } \\
(95 \% \mathrm{CI})\end{array}$ & $\begin{array}{c}\text { IRR } \\
(95 \% \mathrm{Cl})\end{array}$ \\
\hline Overall & $\begin{array}{c}0.09 \\
(0.08-0.11)\end{array}$ & - & $\begin{array}{c}9.26 \\
(8.81-9.73)\end{array}$ & - \\
\hline \multicolumn{5}{|l|}{ By gender } \\
\hline M & $\begin{array}{c}0.06 \\
(0.05-0.08)\end{array}$ & $\begin{array}{c}0.46 \\
(0.35-0.62)\end{array}$ & $\begin{array}{c}7.77 \\
(7.22-8.35)\end{array}$ & $\begin{array}{c}0.70 \\
(0.63-0.77)\end{array}$ \\
\hline $\mathrm{F}$ & $\begin{array}{c}0.14 \\
(0.12-0.16)\end{array}$ & $\begin{array}{l}1.00 \\
(\mathrm{RC})\end{array}$ & $\begin{array}{c}11.08 \\
(10.35-11.86)\end{array}$ & $\begin{array}{l}1.00 \\
(\mathrm{RC})\end{array}$ \\
\hline \multicolumn{5}{|l|}{ By age classes } \\
\hline$<65$ & $\begin{array}{c}0.07 \\
(0.05-0.09)\end{array}$ & $\begin{array}{l}1.00 \\
(\mathrm{RC})\end{array}$ & $\begin{array}{c}8.91 \\
(8.24-9.63)\end{array}$ & $\begin{array}{l}1.00 \\
(\mathrm{RC})\end{array}$ \\
\hline $65-74$ & $\begin{array}{c}0.10 \\
(0.08-0.13)\end{array}$ & $\begin{array}{c}1.50 \\
(1.07-2.11)\end{array}$ & $\begin{array}{c}9.23 \\
(8.52-10.00)\end{array}$ & $\begin{array}{c}1.04 \\
(0.93-1.16)\end{array}$ \\
\hline$>=75$ & $\begin{array}{c}0.15 \\
(0.12-0.20)\end{array}$ & $\begin{array}{c}2.30 \\
(1.60-3.31)\end{array}$ & $\begin{array}{c}9.89 \\
(8.85-11.05)\end{array}$ & $\begin{array}{c}1.11 \\
(0.97-1.27)\end{array}$ \\
\hline \multicolumn{5}{|c|}{ By diabetes duration } \\
\hline$<=2$ & $\begin{array}{c}0.02 \\
(0.01-0.05)\end{array}$ & $\begin{array}{l}1.00 \\
(\mathrm{RC})\end{array}$ & $\begin{array}{c}4.63 \\
(3.75-5.72)\end{array}$ & $\begin{array}{l}1.00 \\
(\mathrm{RC})\end{array}$ \\
\hline $2-5$ & $\begin{array}{c}0.08 \\
(0.06-0.12)\end{array}$ & $\begin{array}{c}3.66 \\
(1.42-9.47)\end{array}$ & $\begin{array}{c}6.63 \\
(5.76-7.63)\end{array}$ & $\begin{array}{c}1.43 \\
(1.11-1.85)\end{array}$ \\
\hline $5.1-10$ & $\begin{array}{c}0.06 \\
(0.04-0.09)\end{array}$ & $\begin{array}{c}2.66 \\
(1.03-6.88)\end{array}$ & $\begin{array}{c}7.38 \\
(6.59-8.27)\end{array}$ & $\begin{array}{c}1.60 \\
(1.26-2.03)\end{array}$ \\
\hline$>10$ & $\begin{array}{c}0.14 \\
(0.12-0.17)\end{array}$ & $\begin{array}{c}6.30 \\
(2.58-15.38)\end{array}$ & $\begin{array}{c}11.90 \\
(11.15-12.69)\end{array}$ & $\begin{array}{c}2.57 \\
(2.06-3.21)\end{array}$ \\
\hline \multicolumn{5}{|c|}{ By cardiac/cerebrovascular disease } \\
\hline NO & $\begin{array}{c}0.10 \\
(0.09-0.12)\end{array}$ & $\begin{array}{c}1.16 \\
(0.79-1.70)\end{array}$ & $\begin{array}{c}8.7 \\
(8.22-9.21)\end{array}$ & $\begin{array}{c}0.74 \\
(0.65-0.83)\end{array}$ \\
\hline YES & $\begin{array}{c}0.09 \\
(0.06-0.12)\end{array}$ & $\begin{array}{l}1.00 \\
(\mathrm{RC})\end{array}$ & $\begin{array}{c}11.83 \\
(10.67-13.13)\end{array}$ & $\begin{array}{l}1.00 \\
(\mathrm{RC})\end{array}$ \\
\hline \multicolumn{5}{|c|}{ By glucose lowering therapy schemes } \\
\hline $\begin{array}{l}\text { OHA (without } \\
\text { secretagogues) }\end{array}$ & $\begin{array}{c}0.04 \\
(0.03-0.06)\end{array}$ & $\begin{array}{r}1.00 \\
(\mathrm{RC})\end{array}$ & $\begin{array}{c}5.57 \\
(4.98-6.22)\end{array}$ & $\begin{array}{r}1.00 \\
(\mathrm{RC})\end{array}$ \\
\hline $\begin{array}{l}\text { OHA (with } \\
\text { secretagogues) }\end{array}$ & $\begin{array}{c}0.08 \\
(0.06-0.11)\end{array}$ & $\begin{array}{c}2.00 \\
(1.24-3.21)\end{array}$ & $\begin{array}{c}9.50 \\
(8.64-10.45)\end{array}$ & $\begin{array}{c}1.71 \\
(1.48-1.98)\end{array}$ \\
\hline $\begin{array}{l}\text { Insulin } \\
\text { (basal oral) }\end{array}$ & $\begin{array}{c}0.10 \\
(0.06-0.17)\end{array}$ & $\begin{array}{c}2.49 \\
(1.31-4.73)\end{array}$ & $\begin{array}{c}10.76 \\
(9.01-12.85)\end{array}$ & $\begin{array}{c}1.93 \\
(1.57-2.38)\end{array}$ \\
\hline $\begin{array}{l}\text { Insulin } \\
\text { (basal bolus) }\end{array}$ & $\begin{array}{c}0.29 \\
(0.23-0.35)\end{array}$ & $\begin{array}{c}6.91 \\
(4.52-10.56)\end{array}$ & $\begin{array}{c}18.36 \\
(16.79-20.08)\end{array}$ & $\begin{array}{c}3.30 \\
(2.86-3.80)\end{array}$ \\
\hline $\begin{array}{l}\text { Insulin } \\
\text { (Other schemes) }\end{array}$ & $\begin{array}{c}0.13 \\
(0.08-0.19)\end{array}$ & $\begin{array}{c}3.02 \\
(1.70-5.36)\end{array}$ & $\begin{array}{c}14.55 \\
(12.64-16.75)\end{array}$ & $\begin{array}{c}2.61 \\
(2.19-3.13)\end{array}$ \\
\hline Diet & 0 & - & $\begin{array}{c}1.60 \\
(1.10-2.34)\end{array}$ & $\begin{array}{c}0.29 \\
(0.19-0.43)\end{array}$ \\
\hline
\end{tabular}

OHA: Oral Hypoglycemic Agents

RC: Reference Class

IR: Incidence Rate

95\% Cl: 95\% Confidence Interval

IRR: Incidence Rate Ratio

Table 2: Incidence of severe and symptomatic hypoglycemia in type 2 diabetes

The risk of severe hypoglycemia was about three times higher in patients with previous severe and symptomatic hypoglycemia; furthermore, a doubled risk of severe episodes was associated with female gender, presence of neoplasms and use of a basal-bolus insulin regimen. Additionally, a $73 \%$ higher risk was found in the presence of sensory-motor neuropathy. The risk increased by $2 \%$ for each unit increase in age and by $2 \%$ for each year of diabetes duration increase. The risk was $80 \%$ higher in employed patients as compared to unemployed ones.

At multivariate analysis, glucose-lowering therapy (secretagogues and insulin) and previous severe hypoglycemia were the strongest predictors of symptomatic hypoglycemia, followed by relevant clinical characteristics, such as diabetes duration, presence of retinopathy, and sensory-motor neuropathy. On the other hand, renal function impairment was associated with a lower likelihood of symptomatic episodes. The number of overall drugs taken by the patient was also related to the risk of hypoglycemia, while the use of beta-blockers was associated with a $16 \%$ lower likelihood to report episodes. Female gender and taking care of other people were also associated with an increased risk of symptomatic episodes.

\section{Discussion}

HYPOS-1 initiative showed for the Italian population with type 2 diabetes followed by DOCs that one in twenty patients experienced at least 1 severe episode and a minority of patients accounted for the vast majority of severe episodes. In terms of disease burden, healthcare 
a. Severe hypoglycemia

\begin{tabular}{|l|c|}
\hline Variables & IRR (95\%Cl) \\
\hline Age & $1.02(1.00-1.04)$ \\
\hline Female gender & $2.23(1.61-3.08)$ \\
\hline Duration of diabetes & $1.02(1.01-1.04)$ \\
\hline HbA1c & $1.28(1.14-1.44)$ \\
\hline Previous severe hypoglycemia (before the past 12 months) & $3.38(2.47-4.62)$ \\
\hline Symptomatic hypoglycemic episodes in the last 4 weeks & $3.05(2.18-4.26)$ \\
\hline Therapeutic schemes & \\
\hline Insulin (Other schemes) & $0.91(0.48-1.74)$ \\
\hline Insulin (basal bolus) & $2.04(1.24-3.35)$ \\
\hline Insulin (basal oral) & $0.79(0.38-1.67)$ \\
\hline OHA (combination with secretagogues) & $1.07(0.64-1.78)$ \\
\hline OHA (combination without secretagogues) & $1.00(\mathrm{RC})$ \\
\hline Sensory-motor neuropathy & $1.73(1.24-2.41)$ \\
\hline Neoplasms & $2.08(1.23-3.49)$ \\
\hline Employment status & \\
\hline Retired & $0.94(0.62-1.43)$ \\
\hline Employed & $1.80(1.08-3.01)$ \\
\hline Unemployed & $1.00(\mathrm{RC})$ \\
\hline
\end{tabular}

\section{b. Symptomatic hypoglycemia}

\begin{tabular}{|l|l|}
\hline Variables & IRR (95\%CI) \\
\hline Age & $0.98(0.97-0.99)$ \\
\hline Female gender & $1.44(1.29-1.62)$ \\
\hline Duration of diabetes & $1.02(1.01-1.02)$ \\
\hline HbA1c & $0.90(0.86-0.95)$ \\
\hline Previous severe hypoglycemia (before the past 12 months) & $1.72(1.51-1.96)$ \\
\hline GFR < 60 mL/min & $0.85(0.74-0.99)$ \\
\hline Retinopathy & $1.25(1.10-1.42)$ \\
\hline Sensory-motor neuropathy & $1.20(1.04-1.39)$ \\
\hline Therapeutic schemes & \\
\hline Insulin (Other schemes) & $2.15(1.75-2.65)$ \\
\hline Insulin (basal bolus) & $2.59(2.18-3.09)$ \\
\hline Insulin (basal oral) & $1.76(1.39-2.23)$ \\
\hline OHA (combination with secretagogues) & $1.63(1.39-1.91)$ \\
\hline OHA (combination without secretagogues) & $1.00(\mathrm{RC})$ \\
\hline Beta-blockers treatment & $0.84(0.73-0.96)$ \\
\hline Number of drugs other than antidiabetic ones & $1.02(1.00-1.05)$ \\
\hline Living status & \\
\hline Other & $1.54(1.17-2.02)$ \\
\hline Other family members & $0.70(0.52-0.95)$ \\
\hline Alone & $1.32(1.15-1.53)$ \\
\hline Spouse/sons & $1.00(\mathrm{RC})$ \\
\hline Taking care of other people & $1.45(1.29-1.62)$ \\
\hline Employment status & \\
\hline Retired & $1.39(1.19-1.63)$ \\
\hline Employed & $1.08(0.89-1.30)$ \\
\hline Unemployed & $1.00(\mathrm{RC})$ \\
\hline RC: Referen & \\
\hline
\end{tabular}

RC: Reference Class

Table 3: Factors associated with risk of hypoglycemia. Results of the Poisson multivariate analysis

Table shows only statistically significant rate ratios (95\% confidence intervals).

system is expected to manage one severe episode every ten patients seen during a year. Symptomatic hypoglycemia was extremely common, affecting over one in four of the sample.

Incidence of hypoglycemia in our study was higher than incidences documented in randomized clinical trials [21]. In particular, in the ACCORD, ADVANCE and VADT trials, designed to test whether treatment targeting nearly normal glycemic control reduces the risk of cardiovascular events in type 2 diabetes, showed incidence rates for severe hypoglycemia in intensive insulin group ranging from 0.7 to 12 events/100 person-years. We found an incidence of severe hypoglycemia ranging from 4 (OHA without secretagogues) to 29 (Insulin - basal bolus) events $/ 100$ person-years.

Incidence of hypoglycemia in type 2 diabetes, especially selftreated one, has been rarely evaluated outside of clinical trial settings, and studies are almost exclusively focused on insulin-treated patients [22]; in addition, data from the real world are extremely heterogeneous due to the differences in the methods of data collection and population selection [23]. In a consecutive sample identified at Steno Diabetes Center consisting of 401 subjects with type 2 diabetes treated with insulin, an overall incidence of severe hypoglycemia of 0.44 episodes/ person year was documented [24]. Data from a Scottish Diabetes register on 173 cases of insulin-treated type 2 diabetes showed a rate of 16.37 non severe events/person-years and a rate of 0.35 severe events/ person-years [25]. A large initiative involving 7 European countries (but not Italy) documented an incidence of non severe hypoglycemia of 20-35 events/person-years according to the different insulin regimens [26]. In our study we found incidence rates for patients treated with insulin of 0.25 events/person-years for severe hypoglycemia and 18.8 events/person-years for symptomatic hypoglycemia.

HYPOS-1 adds important information on the global burden of hypoglycemia in Italian patients attending DOCs.

HYPOS-1 documents that some categories of patients are particularly exposed to the risk of hypoglycemia. After adjusting for socio-demographic and clinical characteristics, several independent correlates of severe and symptomatic hypoglycemia were identified. In line with existing literature [24-26], insulin treatment represents the most important risk factor for severe hypoglycemia. In addition, the risk of major hypoglycemia was significantly higher in patients with previous severe episodes [25]; our study shows that also the incidence of symptomatic hypoglycemia is a strong correlate of severe episodes.

In our study, female gender was associated with a two-fold risk of severe episodes. An increased risk of hypoglycemia in women was previously reported in the ACCORD trial [27], but not in observational studies. The reasons for such an increased risk remain to be elucidated.

We also found that the risk of hypoglycemia was significantly higher in the presence of neoplasms, thus underlying the importance of the overall clinical conditions of the patient in determining his risk profile. The importance of the frailty of the patient is further documented by the association with diabetes complications, increasing age and diabetes duration $[27,28]$. Older age and longer diabetes duration, together with diabetes neuropathy, can also represent proxy indicators for hypoglycemia unawareness, a well known risk factor for severe hypoglycemia $[29,30]$.

In previous studies, being married and low levels of school education were identified as correlates of hypoglycemia [24,27]. We could not confirm an association of socio-economic characteristics with the risk of severe hypoglycemia, with the only exception of a higher risk in employed individuals as compared with unemployed ones. Unfortunately, we have no additional information to evaluate whether specific types of employment confer an excess risk of hypoglycemia.

Risk of severe hypoglycemia increased by $28 \%$ for each unit increase 
in HbAlc levels. This point deserves consideration. Higher levels of $\mathrm{HbA} 1 \mathrm{c}$ in patients experiencing severe hypoglycemia could be the result of compensatory behaviors, less aggressive therapeutic approaches, and/or a proxy of glycaemic variability, previously advocated as having an independent role in determining individual's risk of hypoglycemia [31,32].

Correlates of symptomatic hypoglycemia have been seldom investigated, mainly in dated studies. In a study conducted in 1991 the frequency of hypoglycemia was positively, but only weakly, correlated with insulin therapy, HbAlc and age [33]. In another study conducted in 2001 [34] insulin therapy, lower HbA1c level at follow-up, younger age, and report of hypoglycemia at the baseline visit were independently associated with increased prevalence of hypoglycemia.

Our study confirms an inverse relationship between the risk of symptomatic hypoglycemia and age and HbAlc levels, as well as the excess risk associated with sulphonylureas and insulin therapy. In addition, female gender, increasing diabetes duration, previous severe hypoglycemia, the presence of eye and neuropathic complications and increasing number of drugs other than glucose-lowering ones are associated with a higher likelihood of symptomatic hypoglycemia. On the other hand, a low GFR was associated with a lower risk of hypoglycemia, suggesting that a greater attention is devoted in choosing treatment options in individuals with impaired renal function.

The use of beta-blockers is associated with a $16 \%$ lower likelihood of symptomatic hypoglycemia, in line with existing evidence of impaired awareness associated with the use of this class of medications [35].

The significant correlation among different socio-demographic aspects and risk of symptomatic episodes document the importance of daily life and family support in determining an adequate selfmanagement of a chronic disease [36].

Effective approaches identified by a recent ADA consensus to decrease the risk of iatrogenic hypoglycemia include "patient education, dietary and exercise modifications, medication adjustment, careful glucose monitoring by the patient, and conscientious surveillance by the clinician" [37]. Among the current knowledge gaps, it is recognized the difficulty in understanding which patients are most at risk for hypoglycemia.

HYPOS-1 study enlarges the chance to identify patients at increased risk of both severe and symptomatic episodes.

Study results will be used by the AMD scientific society to implement new strategies for promoting a more appropriate use of glucose lowering drugs and for acting on modifiable factors which play a role in the hypoglycemia burden.

The study has strengths and limitations to be discussed. Main strengths are: the evaluation of the impact of both severe and symptomatic hypoglycemia; the inclusion in the analysis of a sample representative of the whole population with type 2 diabetes and not only of those treated with insulin, and the availability of a large number of socio-demographic and clinical variables to be correlated with the risk of hypoglycemia and usually lacking in similar studies. The study also has limitations, including the retrospective design and the self-reported information on symptomatic hypoglycemia, not confirmed by blood glucose self-monitoring values. Furthermore, we used as correlates of hypoglycemia the last available value of $\mathrm{HbAlc}$ and serum creatinine. As such, these measures could be posterior to the major hypoglycemic event, and may not strictly reflect the situation of the patients when they experienced the event. Finally, the role of glycaemic variability as a correlate of the risk of hypoglycemia has been underlined in several studies [38-40]. We could not assess its role due to the lack of information on SMBG.

In conclusion, our study provides an up to date and comprehensive assessment of the incidence and factors associated with the risk of hypoglycemia in everyday clinical practice. Despite the increasing recognition of the risks associated with both severe and symptomatic episodes, hypoglycemia still represents a common acute complication for individuals with type 2 diabetes. Greater attention should be devoted to the choice of individualized targets and treatment schemes, as well as better self-management education, in order to minimize the risk of such a complication, while ensuring adequate metabolic control.

\section{Acknowledgments}

The authors thank the participating diabetes outpatient centers and the whole study staff for their contribution. The authors acknowledge NovoNordisk SpA, Italy for having supported this study. The sponsor did not participate in the design or conduct of this study; in the collection, management, analysis, or interpretation of data; in the writing of the manuscript; or in the preparation, review, approval, or decision to submit this manuscript for publication. Dr. Carlo Giorda, is the guarantor of this work and, as such, had full access to all the data in the study and takes responsibility for the integrity of the data and the accuracy of the data analysis.

\section{Contributors}

All authors were involved in the concept and design of the initiative. GL and MCR performed data analysis. G.C., A.N. and M.C.R. wrote the manuscript. A.O. S.G., A.C., R.I. and F.B. were involved in critical revision for intellectual content and interpretation of data. All authors were involved in the final approval of the manuscript to be submitted for publication.

\section{Funding}

Study supported by an unrestricted grant of NovoNordisk SpA, Rome, Italy

\section{Competing interests}

C.B.G. is a member of the advisory board of Novo Nordisk and Bristol- Myers Squibb; he has received speaker fees from Astrazeneca, Boehringer Ingelheim Bruno Farmaceutici, Novo Nordisk and Bristol- Myers Squibb. S.G. is a member of the advisory board of Sanofi Aventis. A.N. is a member of the advisory board of Novo Nordisk, Novartis, Merk Sharp \& Dohme; he has received speaker fees from Novo Nordisk, Novartis, Merk Sharp \& Dohme. Other authors declare that they have no conflict of interest.

\section{References}

1. Holman RR, Paul SK, Bethel MA, Matthews DR, Neil HA (2008) 10-year followup of intensive glucose control in type 2 diabetes. N Engl J Med 359: 15771589.

2. Action to Control Cardiovascular Risk in Diabetes Study Group, Gerstein HC Miller ME, Byington RP, Goff DC Jr, et al. (2008) Effects of intensive glucose lowering in type 2 diabetes. N Engl J Med 358: 2545-2559.

3. Cryer PE (2008) The barrier of hypoglycemia in diabetes. Diabetes 57: 3169 3176.

4. Goto A, Arah OA, Goto M, Terauchi Y, Noda M (2013) Severe hypoglycaemia and cardiovascular disease: systematic review and meta-analysis with bias analysis. BMJ 347: $\mathrm{f} 4533$.

5. Whitmer RA, Karter AJ, Yaffe K, Quesenberry CP Jr, Selby JV (2009) Hypoglycemic episodes and risk of dementia in older patients with type 2 diabetes mellitus. JAMA 301: 1565-1572.

6. Yaffe K, Falvey CM, Hamilton N, Harris TB, Simonsick EM, et al. (2013) Association between hypoglycemia and dementia in a biracial cohort of older adults with diabetes mellitus. JAMA Intern Med 173: 1300-1306.

7. Hsu PF, Sung SH, Cheng HM, Yeh JS, Liu WL, et al. (2013) Association of clinical symptomatic hypoglycemia with cardiovascular events and total mortality in type 2 diabetes: a nationwide population-based study. Diabetes Care 36: 894-900.

8. Barendse S, Singh H, Frier BM, Speight J (2012) The impact of hypoglycaemia on quality of life and related patient-reported outcomes in Type 2 diabetes: a narrative review. Diabet Med 29: 293-302. 
Citation: Giorda CB, Ozzello A, Gentile S, Corsi A, lannarelli R, et al. (2014) Incidence and Correlates of Hypoglycemia in Type 2 Diabetes. The Hypos-1 Study. J Diabetes Metab 5: 344. doi:10.4172/2155-6156.1000344

9. Nicolucci A, Cucinotta D, Squatrito S, Lapolla A, Musacchio N, et al. (2009) Clinical and socio-economic correlates of quality of life and treatment satisfaction in patients with type 2 diabetes. Nutr Metab Cardiovasc Dis 19: $45-53$

10. Graveling AJ, Frier BM (2009) Hypoglycaemia: an overview. Prim Care Diabetes 3: 131-139.

11. Workgroup on Hypoglycemia, American Diabetes Association (2005) Defining and reporting hypoglycemia in diabetes: a report from the American Diabetes Association Workgroup on Hypoglycemia. Diabetes Care 28: 1245-1249.

12. Barnett AH, Cradock S, Fisher M, Hall G, Hughes E, et al. (2010) Key considerations around the risks and consequences of hypoglycaemia in people with type 2 diabetes. Int J Clin Pract 64: 1121-1129.

13. Leiter LA, Yale J-F, Chiasson J-L, Harris S, Kleinstiver P, Sauriol L (2005) Assessment of the impact of fear of hypoglycemic episodes on glycemic and hypoglycemia management. Can J Diabetes 29: 186-192.

14. Amiel SA, Dixon T, Mann R, Jameson K (2008) Hypoglycaemia in Type 2 diabetes. Diabet Med 25: 245-254.

15. Leese GP, Wang J, Broomhall J, Kelly P, Marsden A, et al. (2003) Frequency of severe hypoglycemia requiring emergency treatment in type 1 and type 2 diabetes: a population-based study of health service resource use. Diabetes Care 26: 1176-1180

16. Willis WD, Diago-Cabezudo JI, Madec-Hily A, Aslam A (2013) Medical resource use, disturbance of daily life and burden of hypoglycemia in insulin-treated patients with diabetes: results from a European online survey. Expert Rev Pharmacoecon Outcomes Res 13: 123-130.

17. The AMD Annals 2010 Working Group, Cimino A, Fava D, Giorda CB Meloncelli I, Nicolucci A (2011) Quality Indicators in Diabetes Care in Italy. AMD Assiociazione Medici Diabetologi.

18. Signorini DF (1991) Sample size for Poisson regression. Biometrika 78: 446450.

19. Pedersen-Bjergaard $U$, Agerholm-Larsen $B$, Pramming $S$, Hougaard $P$ Thorsteinsson B (2001) Activity of angiotensin-converting enzyme and risk of severe hypoglycaemia in type 1 diabetes mellitus. Lancet 357: 1248-1253.

20. Mills GA, Horn JR (1985) Beta-blockers and glucose control. Drug Intell Clin Pharm 19: 246-251.

21. UK Hypoglycaemia Study Group (2007) Risk of hypoglycaemia in types 1 and 2 diabetes: effects of treatment modalities and their duration. Diabetologia 50 : 1140-1147.

22. Desouza CV, Bolli GB, Fonseca V (2010) Hypoglycemia, diabetes, and cardiovascular events. Diabetes Care 33: 1389-1394.

23. Akram K, Pedersen-Bjergaard U, Borch-Johnsen K, Thorsteinsson B (2006) Frequency and risk factors of severe hypoglycemia in insulin-treated type 2 diabetes: a literature survey. J Diabetes Complications 20: 402-408.

24. Akram K, Pedersen-Bjergaard U, Carstensen B, Borch-Johnsen $K$ Thorsteinsson B (2006) Frequency and risk factors of severe hypoglycaemia in insulin-treated Type 2 diabetes: a cross-sectional survey. Diabet Med 23: 750-756.

25. Donnelly LA, Morris AD, Frier BM, Ellis JD, Donnan PT, et al. (2005) Frequency and predictors of hypoglycaemia in Type 1 and insulin-treated Type 2 diabetes: a population-based study. Diabet Med 22: 749-755.
26. Östenson CG, Geelhoed-Duijvestijn P, Lahtela J, Weitgasser R, Markert Jensen M, et al. (2014) Self-reported non-severe hypoglycaemic events in Europe. Diabet Med 31: 92-101.

27. Miller ME, Bonds DE, Gerstein HC, Seaquist ER, Bergenstal RM, et al. (2010) The effects of baseline characteristics, glycaemia treatment approach, and glycated haemoglobin concentration on the risk of severe hypoglycaemia: post hoc epidemiological analysis of the ACCORD study. BMJ 340: b5444.

28. Boucai L, Southern WN, Zonszein J (2011) Hypoglycemia-associated mortality is not drug-associated but linked to comorbidities. Am J Med 124: 1028-1035.

29. Zammitt NN, Frier BM (2005) Hypoglycemia in type 2 diabetes: pathophysiology, frequency, and effects of different treatment modalities. Diabetes Care 28 2948-2961.

30. Henderson JN, Allen KV, Deary IJ, Frier BM (2003) Hypoglycaemia in insulintreated Type 2 diabetes: frequency, symptoms and impaired awareness. Diabet Med 20: 1016-1021.

31. Kilpatrick ES, Rigby AS, Goode K, Atkin SL (2007) Relating mean blood glucose and glucose variability to the risk of multiple episodes of hypoglycaemia in type 1 diabetes. Diabetologia 50: 2553-2561.

32. Lipska KJ, Warton EM, Huang ES, Moffet HH, Inzucchi SE, et al. (2013) HbA1c and risk of severe hypoglycemia in type 2 diabetes: the Diabetes and Aging Study. Diabetes Care 36: 3535-3542.

33. Pramming S, Thorsteinsson B, Bendtson I, Binder C (1991) Symptomatic hypoglycaemia in 411 type 1 diabetic patients. Diabet Med 8: 217-222.

34. Miller CD, Phillips LS, Ziemer DC, Gallina DL, Cook CB, El-Kebbi IM (2001) Hypoglycemia in patients with type 2 diabetes mellitus. Arch Intern Med 161 1653-1659.

35. Schwab KO, Menche U, Schmeisl G, Lohse MJ (2004) Hypoglycemiadependent beta2-adrenoceptor downregulation: a contributing factor to hypoglycemia unawareness in patients with Type-1 diabetes? Horm Res 62 137-141.

36. Nicolucci A, Kovacs Burns K, Holt RI, Comaschi M, Hermanns N, et al. (2013) Diabetes attitudes, wishes and needs second study (DAWN2 ${ }^{\mathrm{TM}}$ ): cross-national benchmarking of diabetes-related psychosocial outcomes for people with diabetes. Diabet Med 30: 767-777.

37. Seaquist ER, Anderson J, Childs B, Cryer P, Dagogo-Jack S, et al. (2013) Hypoglycemia and diabetes: a report of a workgroup of the American Diabetes Association and the Endocrine Society. Diabetes Care 36: 1384-1395

38. Kovatchev BP, Otto E, Cox D, Gonder-Frederick L, Clarke W (2006) Evaluation of a new measure of blood glucose variability in diabetes. Diabetes Care 29 : 2433-2438.

39. Monnier L, Wojtusciszyn A, Colette C, Owens D (2011) The contribution of glucose variability to asymptomatic hypoglycemia in persons with type 2 diabetes. Diabetes Technol Ther 13: 813-818.

40. Rodbard D (2012) Hypo- and hyperglycemia in relation to the mean, standard deviation, coefficient of variation, and nature of the glucose distribution. Diabetes Technol Ther 14: 868-876. 